\title{
Calidad de vida y necesidad de tratamiento ortodóncico en entidades educativas de Huancayo, Perú 2020
}

\author{
Quality of life and need for orthodontic treatment in educational institutions of Huancayo, Peru 2020
}

Pablo Santiago Bonilla Cairo ${ }^{1, a}$, Luis Alberto Cueva Buendía ${ }^{1, b}$, Evelyn Jeannet Jesús Balbín 1,c

\section{RESUMEN}

Objetivo: determinar la relación entre la calidad de vida mediante el COHIP 19 SF y la necesidad de tratamiento ortodóntico mediante el componente CE del INTO en escolares de 12 a 15 años de dos entidades educativas pública y privada de Huancayo 2020. Material y Métodos: Estudio descriptivo, transversal y comparativo se evaluaron a 318 escolares de dos unidades educativas, pública y privada. Para evaluar el nivel de necesidad de tratamiento ortodóntico se utilizó el Índice de Necesidad de Tratamiento de Ortodoncia (INTO -CE) y para la calidad de vida se empleó el índice de Perfil de Impacto en Salud Oral Infantil en su versión corta (COHIP SF-19), el análisis bivariado se llevó a cabo mediante la Prueba de Rho de Spearman y se consideró un nivel de significancia de 0,05 . Resultados: De acuerdo al componente estético (CE) del INTO, la necesidad definitiva fue 5,3\%, necesidad moderada de 4,4\%, y el $90.3 \%$ sin necesidad de tratamiento ortodóntico. La calidad de vida tuvo una media de 54.3, para el bienestar social y emocional su media fue del 27.1, el bienestar funcional de 12.5 y la salud oral fue de 14.6. Conclusiones: Existe correlación negativa moderada entre la calidad de vida y la necesidad de tratamiento ortodóntico (CE) en escolares de 12 a 15 años de dos unidades educativas público y privada de la ciudad de Huancayo 2020, la calidad de vida fue baja siendo mayor en las mujeres que los varones con diferencia estadística significativa (Rho - 0,635 ; p-valor 0,023$)$.

PALABRAS CLAVE: Calidad de vida, necesidad de tratamiento ortodóncico.

\section{SUMMARY}

Objective: to determine the relationship between quality of life through COHIP 19 SF and the need for orthodontic treatment through the CE component of INTO in schoolchildren aged 12 to 15 years from two public and private educational entities in Huancayo 2020. Methodology Descriptive, cross-sectional study and comparative, 318 schoolchildren from two educational units, public and private, were evaluated. To assess the level of need for orthodontic treatment, the Orthodontic Treatment Need Index (INTO -CE) was used and for quality of life the Impact Profile index on Children's Oral Health was used in its short version (COHIP SF- 19), the bivariate analysis was carried out using the Spearman Rho Test and a significance level of 0.05 was considered. Results: According to the aesthetic component (CE) of the INTO, the definitive need was $5.3 \%$, moderate need $4.4 \%$, and $90.3 \%$ without the need for orthodontic treatment. The quality of life had an average of 54.3, for social and emotional well-being its average was 27.1, functional well-being of 12.5 and oral health was 14.6. Conclusions: There is a moderate

\footnotetext{
Universidad Privada de Huancayo Franklin Roosevelt. Huancayo, Perú.

Docente; Cirujano dentista; Doctor. ORCID ID: 0000-0001-8412-6521

Docente; Cirujano dentista; Magister. ORCID ID: 0000-0001-5003-7352

Docente; Magister; Lic. en Psicología.ORCID ID: 0000-0002-1846-5936
} 
negative correlation between quality of life and the need for orthodontic treatment (CE) in schoolchildren aged 12 to 15 years from two public and private educational units in the city of Huancayo 2020, the quality of life was low, being higher in women than men with statistically significant difference (Rho -0.635; p-value 0.023).

KEY WORDS: Quality of life and need for orthodontic treatment

\section{INTRODUCCIÓN}

La maloclusión es la tercera patología oral de mayor prevalencia a nivel mundial según la Organización Mundial de la Salud (OMS), esta alteración morfológica compromete a cualquier componente de nuestro sistema estomatognático y puede ocasionar problemas psicosociales, funcionales o predisponer al padecimiento de algunas otras patologías como son la caries dental, problemas periodontales, de la articulación temporomandibular así como predisponer a traumatismos. Para los ortodoncistas el objetivo de tratamiento como la oclusión dental definida habitualmente como el contacto estático de los dientes en oclusión ha sido reemplazada ampliamente por el aspecto facial y la exposición de los dientes, sabemos que en la interacción social muchas veces se valora más el aspecto facial que el comportamiento, deseando resultar agradables a otros, por lo tanto destacamos y buscamos un tratamiento que ayude a superar o minimizar un obstáculo social que disminuya la calidad de vida (1). La definición de calidad de vida es muy amplia de acuerdo con la óptica en que se la mire, sin embargo, desde un punto de vista de salud oral, es definida como "Una cavidad oral en condiciones de salud que le permita al individuo comer, hablar y socializar libre de dolor, enfermedad o avergonzamiento social", cualquier afectación debe ser lo suficiente para afectar la calidad de vida, definitivamente la maloclusión favorece a ello. El grupo etario del adolescente es particularmente más sensible a generar problemas psicológicos y un bajo nivel en su calidad de vida, debido a que sabemos según la psicología infantil que a partir de los 6 años la autopercepción ya es concebida, a los 10 años ya tiene una conceptualización de su estética y entre los 11 y 14 es tangible el impacto de la autopercepción estética en su vida (2). Para valorar la maloclusión se tiene muchos índices, dentro de ellos uno muy utilizado es de INTO (índice de necesidad de tratamiento), tiene dos componentes uno el CSD (componente de salud dental) que lo aplica el profesional y otro que es en base a 10 fotografías presentadas al paciente y por su autopercepción relaciona el que más se acerque a su condición estética (CE); para medir la calidad de vida relacionada con la salud bucal (CVRO) existen igualmente muchos, uno de ellos es el COHIP-SF 19 especialmente diseñado para medir el CVRO en adolescentes, que ha demostrado ser útil en muchos estudios para determinar el impacto de las maloclusiones en la calidad de vida de los adolescentes. Aunque las enfermedades bucales comunes no ponen en peligro la vida, pueden influir en el bienestar general de las personas. Esto ha resultado en un mayor enfoque clínico en la mejora de la calidad de vida como un objetivo principal del cuidado dental para afecciones como problemas de ortodoncia (3).

En un estudio realizado por Feiou et al, en 2018 relaciona el comportamiento psicológico del paciente y calidad de vida con respecto a la maloclusión, refieren que pacientes con maloclusión son más propensos a ser introvertidos e inestables que los individuos con oclusión normal (12) (4). Mientras tanto, la autoestima relacionada con la apariencia facial tiende a correlacionarse estrechamente con la personalidad, ya que la insatisfacción con la apariencia dental es un fuerte predictor de baja autoestima. Estos hallazgos indican que la gravedad de la maloclusión está asociada con la calidad de vida de las personas (4).

Crespo C. et al. Evalúan el impacto de las condiciones orales atribuidas a maloclusiones sobre la calidad de vida a través del CS Child-OIDP y la necesidad de tratamiento ortodóntico a través del INTO en 170 escolares de 11 a 12 años de dos escuelas privadas en Azogues-Ecuador, evalúan la influencia de factores como posición de los dientes, espacios, tamaño, forma y deformidades craneofaciales, sobre los desempeños diarios; compararon la necesidad de tratamiento ortodóntico evaluado con el INTO; obtienen como resultado que las condiciones más prevalentes fueron: posición de los dientes $(63,5 \%)$, y dientes separados $(40,5 \%)$; los desempeños más afectados fueron comer $(65,3 \%)$ y sonreír $(51,8 \%)$; respecto de la necesidad de tratamiento ortodóntico según el componente estético, el 91,18\% de los 
escolares no tuvieron necesidad de tratamiento y según el componente de salud dental, el 10\%; al asociar las dos variables, se encontró diferencia estadísticamente significativa en el desempeño sonreír $\mathrm{p}=0,02$; según el Componente de Salud Dental, llegando a la conclusión que la condición más prevalente fue posición de los dientes, los desempeños diarios más afectados fueron comer y sonreír (5).

Campoverde en un estudio realizado en Ecuador el 2016 evaluó la necesidad de tratamiento Ortodóntico a través del INTO y su relación con la calidad de vida relacionada a la salud oral a través del COHIPSF 19 en escolares de 12 a 15 años de dos unidades educativas, pública y privada de la ciudad de LojaEcuador, encuentró que, según el CSD, la necesidad definitiva fue del $66,66 \%$, necesidad moderada del $10,42 \%$, y sin necesidad el 22,92\% (8)(2). Según el CE, la necesidad definitiva fue del $10,42 \%$, necesidad moderada de $8,33 \%$, y el $81,30 \%$ sin necesidad. La calidad de vida tuvo una media de 53,65 (D.E. $=10,39$ ), para el bienestar social y emocional su media fue del $=28,77$ (D.E. $=6,15$ ), el bienestar funcional de $=12,01$ (D.E. $=2,87)$ y la salud oral $=12.87$ (D.E. $=3,53$ ). Concluyendo que la necesidad clínica de tratamiento Ortodóntico es alta, afectando la Calidad de vida cuyo promedio es bajo. La necesidad de tratamiento fue mayor en el colegio público y en el sexo masculino. La calidad de vida fue más alta en el colegio público y en el sexo masculino (2).

El objetivo de este trabajo fue determinar la relación entre la calidad de vida mediante el COHIP 19 SF y la necesidad de tratamiento ortodóntico mediante el componente CE del INTO en escolares de 12 a 15 años de dos entidades educativas pública y privada de Huancayo 2020.

\section{MATERIAL Y METODOS}

El presente estudio corresponde a un diseño descriptivo y transversal, la población estuvo conformada por 700 estudiantes entre 12 a 15 años de dos escuelas privadas de la ciudad de HuancayoPerú, cuya muestra final quedó conformada por 318 individuos; siendo 258 de colegio público y 60 de colegio privado, hallado a través del muestreo aleatorio, en el estudio se incluyeron todos los escolares del rango de edad establecido cuyos padres firmaron el consentimiento informado y además los estudiantes dieron su asentimiento, excluyéndose del estudio a quienes no lo hicieron y se encontraban bajo tratamientos de Ortodoncia, deformidades dentofaciales y síndromes diagnosticados. Se utilizó una entrevista estructurada para la aplicación del COHIP-SF 19, y la escala estética del INTO. Se obtuvo la aprobación del Comité Institucional de Ética de la Universidad Franklin Roosevelt, así como las autorizaciones respectivas de las autoridades educativas. El proceso de recolección de datos cumplió en dos etapas, primero se obtuvo la información de los datos generales, luego se realizó la entrevista (COHIPSF 19) conjuntamente con el componente CE del INTO con la selección de una fotografía ; el índice se aplicó a través de entrevistas individuales a través del whatsap con previa autorización de los padres de familia, para obtener información acerca de la calidad de vida del paciente se hizo un listado de 19 preguntas las cuales están comprendidas en tres grupos, la Salud oral preguntas: 1 a 5 . Bienestar funcional preguntas: $9,13,17,18$. Bienestar emocional y social preguntas: $6,7,8,10,11,12,14,15,16,19$. De las 19 preguntas, 17 evalúan aspectos negativos relacionados con la salud oral del paciente en una escala descendente de 4 a 0 en donde 4 es nunca, 3 casi nunca, 2 a veces, 1 con frecuencia, 0 casi todo el tiempo. Solamente las preguntas 8 ( $¿$ Te has sentido seguro de ti mismo debido a tus dientes, boca o cara?) y 15 (¿Sientes que eres bonita/guapo o buen mozo?) que abordan aspectos positivos se evalúan en la misma escala, pero de manera inversa 0 a 4 en donde 0 es nunca, 1 casi nunca, 2 a veces, 3 con frecuencia, 4 casi todo el tiempo. Finalmente se sumaron los valores obtenidos y se obtuvo una media, que determinó el promedio de la calidad de vida de la muestra, los valores más altos a la media indicaron una buena calidad de vida, y los que estén por debajo indicaron una mala calidad de vida. En la siguiente etapa se evaluó a los niños a través del INTO, para determinar el CE-INTO se presentaron 10 fotografías intraorales ordenadas desde la 1 hasta la 10 en dos columnas, donde la número 1 estaba catalogada como la mejor y la 10 la peor; se le pidió al niño que busque la foto de acuerdo a la percepción de su estética dental, si la foto elegida se encontraba entre la 1 y la 4, se considera sin necesidad de tratamiento; entre la 5 y 7 , con necesidad moderada de tratamiento; y entre la 8 y 10 , con necesidad urgente de tratamiento, estas fotografías pertenecen a un niño de 12 años.

El análisis bivariado se llevó a cabo mediante la Prueba de Rho de Spearman. Se utilizaron los programas Excel y SPSS v. 23. El estudio contó con un nivel de confianza de $95 \%$ y un nivel de significancia del 0,05 . 


\section{RESULTADOS}

Los resultados obtenidos se muestran en las tablas $1,2,3,4,5,6,7,8$ y 9 .

Tabla 1. Variable necesidad de tratamiento ortodoncico

\begin{tabular}{llcccc}
\hline & Frecuencia & Porcentaje & Porcentaje válido & Porcentaje acumulado \\
\hline \multirow{4}{*}{ Válido } & Sin necesidad & 287 & 90,3 & 90,3 & 90,3 \\
& Necesidad moderada & 14 & 4,4 & 4,4 & 94,7 \\
& Necesidad definitiva & 17 & 5,3 & 5,3 & 100,0 \\
& Total & 318 & 100,0 & 100,0 & \\
\hline
\end{tabular}

Tabla 2. Variable necesidad de tratamiento ortodóncico, según centro educativo

\begin{tabular}{|c|c|c|c|c|c|c|c|}
\hline \multirow{2}{*}{$\begin{array}{l}\text { Variable necesidad de } \\
\text { tratamiento ortodoncico }\end{array}$} & \multicolumn{4}{|c|}{ CENTRO EDUCATIVO } & \multirow{2}{*}{\multicolumn{2}{|c|}{ Total }} & \multirow{3}{*}{ p-valor } \\
\hline & \multicolumn{2}{|c|}{ Estatal } & \multicolumn{2}{|c|}{ Particular } & & & \\
\hline Sin necesidad & 234 & $73,6 \%$ & 53 & $16,7 \%$ & 287 & $90,3 \%$ & \\
\hline Necesidad moderada & 10 & $3,1 \%$ & 4 & $1,3 \%$ & 14 & $4,4 \%$ & \\
\hline Necesidad definitiva & 14 & $4,4 \%$ & 3 & $0,9 \%$ & 17 & $5,3 \%$ &, 078 \\
\hline Total & 258 & $81,1 \%$ & 60 & $18,9 \%$ & 318 & $100 \%$ & \\
\hline
\end{tabular}

Tabla 3. Variable necesidad de tratamiento ortodóncico, según sexo

\begin{tabular}{|c|c|c|c|c|c|c|c|c|c|c|}
\hline \multirow{4}{*}{ SEXO } & & \multicolumn{6}{|c|}{$\begin{array}{c}\text { Variable necesidad de tratamiento } \\
\text { ortodoncico }\end{array}$} & \multirow{2}{*}{\multicolumn{2}{|c|}{ Total }} & \multirow[t]{3}{*}{ p-valor } \\
\hline & \multirow{3}{*}{$\begin{array}{l}\text { Femenino } \\
\text { Masculino }\end{array}$} & \multicolumn{2}{|c|}{$\begin{array}{c}\text { Sin } \\
\text { necesidad }\end{array}$} & \multicolumn{2}{|c|}{$\begin{array}{l}\text { Necesidad } \\
\text { moderada }\end{array}$} & \multicolumn{2}{|c|}{$\begin{array}{c}\text { Necesidad } \\
\text { definitiva }\end{array}$} & & & \\
\hline & & 169 & $53,1 \%$ & 8 & $2,5 \%$ & 9 & $2,8 \%$ & \multirow{2}{*}{$\begin{array}{l}186 \\
132\end{array}$} & \multirow{2}{*}{$\begin{array}{l}58,5 \% \\
41,5 \%\end{array}$} & \\
\hline & & 118 & $37,1 \%$ & 6 & $1,9 \%$ & 8 & $2,5 \%$ & & & 0,62 \\
\hline Total & & 287 & $90,3 \%$ & 14 & $4,4 \%$ & 17 & $5,3 \%$ & 318 & $100,0 \%$ & \\
\hline
\end{tabular}

Tabla 4. Variable necesidad de tratamiento ortodóncico, según edad

\begin{tabular}{lllllllllll}
\hline & & \multicolumn{2}{c}{$\begin{array}{c}\text { No hay } \\
\text { necesidad }\end{array}$} & \multicolumn{2}{c}{$\begin{array}{c}\text { Necesidad } \\
\text { moderada }\end{array}$} & $\begin{array}{c}\text { Necesidad } \\
\text { definitiva }\end{array}$ & Total & p-valor \\
\hline \multirow{2}{*}{ EDAD } & $<$ de 13 años & 184 & $57,9 \%$ & 9 & $2,8 \%$ & 10 & $3,1 \%$ & 203 & $63,8 \%$ & \\
& $\geq$ de 13 años & 103 & $32,4 \%$ & 5 & $1,6 \%$ & 7 & $2,2 \%$ & 115 & $36,2 \%$ & 0,069 \\
& & 287 & $90,3 \%$ & 14 & $4,4 \%$ & 17 & $5,3 \%$ & 318 & $100,0 \%$ & \\
\hline
\end{tabular}

Tabla 5. Variable calidad de vida relacionada a la salud oral

\begin{tabular}{cccccc}
\hline & & Frecuencia & Porcentaje & Porcentaje válido & Media \\
\hline \multirow{4}{*}{ Válido } & Baja & 160 & 50,3 & 50,3 & \\
& Alta & 158 & 49,7 & 49,7 & 54,2 \\
& Total & 318 & 100,0 & 100,0 & \\
\hline
\end{tabular}

Tabla 6. Dimensión salud oral de Calidad de vida

\begin{tabular}{cccccc}
\hline & & Frecuencia & Porcentaje & Porcentaje válido & Media \\
\hline \multirow{3}{*}{ Válido } & Baja & 154 & 48,4 & 48,4 & \\
& Alta & 164 & 51,6 & 51,6 & 14,6 \\
& Total & 318 & 100,0 & 100,0 & \\
\hline
\end{tabular}


Tabla 7. Variable calidad de vida según sexo

\begin{tabular}{llcccc}
\hline & & Baja & Alta & Total & p-valor \\
\hline \multirow{2}{*}{ SEXO } & Femenino & 105 & 81 & 186 & \\
& Masculino & 55 & 77 & 132 & 0,009 \\
Total & & 160 & 158 & 318 & \\
\hline
\end{tabular}

Tabla 8. Variable calidad de vida según centro educativo

\begin{tabular}{lccccc}
\hline & & \multicolumn{2}{c}{ CENTRO EDUCATIVO } & \multirow{2}{*}{ Total } & \multirow{2}{*}{ p-valor } \\
\cline { 2 - 4 } & & Estatal & Particular & & \\
\hline Variable calidad de vida & Baja & 131 & 29 & 160 & \multirow{2}{*}{0,73} \\
relacionada a la salud oral & Alta & 127 & 31 & 158 & \multirow{2}{*}{ Total } \\
\hline
\end{tabular}

Tabla 9. Correlación entre la Variable calidad de vida y necesidad de tratamiento

\begin{tabular}{|c|c|c|c|c|c|}
\hline & & & $\begin{array}{c}\text { VARIABLE } \\
\text { CALIDAD } \\
\text { DE VIDA } \\
\text { RELACIONADAA } \\
\text { LA SALUD ORAL } \\
\end{array}$ & $\begin{array}{c}\text { VARIABLE } \\
\text { NECESIDAD DE } \\
\text { TRATAMIENTO } \\
\text { ORTODONCICO }\end{array}$ & p-valor \\
\hline \multirow{6}{*}{$\begin{array}{l}\text { Rho de } \\
\text { Spearman }\end{array}$} & \multirow{3}{*}{$\begin{array}{l}\text { Variable calidad de } \\
\text { vida relacionada a } \\
\text { la salud oral }\end{array}$} & Coeficiente de correlación & 1,000 &,- 635 & \multirow{6}{*}{0,023} \\
\hline & & Sig. (bilateral) & . &, 016 & \\
\hline & & $\mathrm{N}$ & 318 & 318 & \\
\hline & \multirow{3}{*}{$\begin{array}{l}\text { Variable necesidad } \\
\text { de tratamiento } \\
\text { ortodoncico }\end{array}$} & Coeficiente de correlación &,- 635 & 1,000 & \\
\hline & & Sig. (bilateral) &, 016 & . & \\
\hline & & $\mathrm{N}$ & 318 & 318 & \\
\hline
\end{tabular}

\section{DISCUSIÓN}

El estudio se realizó en la región Junín, provincia de Huancayo, que se encuentra en la región central de Perú que está a una altitud de 3250 m.s.n.m., cuya grupo étnico es de raza mestiza homogénea, con una población de más de 800 mil habitantes según el censo de 2017; se seleccionaron dos grupos de instituciones educativas una pública y otra privada; se consideró el grupo etario de 12 a 15 años, haciendo un total de 318 estudiantes que cumplieron con los criterios de selección, específicamente lo pertinente a no haber recibido o estar con tratamiento ortodóntico u ortopédico, criterios similares lo aplicaron los estudios de Crespo, Campoverde y otros, cabe señalar que es un grupo vulnerable en contraer problemas psicológicos y sociales ya que están en desarrollo y maduración de su personalidad y algún problema como la maloclusión o alteración de su estética facial puede ser motivo de alguna alteración, el análisis para determinar la necesidad de tratamiento INTO se compone de dos subunidades, el empleado en el presente estudio fue el CE (componente estético), quiere decir por una autovaloración de la estética bucal mediante fotografías como se explicó anteriormente, tiene también el componente CS, el cual hace una valoración de la necesidad de tratamiento ortodóntico por parte del profesional mediante un examen clínico; por motivos de la pandemia y emergencia sanitaria en nuestro país se hizo mediante el CE, que como en muchos otros estudios lo emplearon y demostraron su eficacia; la manera de medir la calidad de vida es el COHIP-SF-19 de utilización ampliamente difundida y eficacia comprobada en muchos estudios en el mundo. Al analizar el INTO CE, se puede apreciar un alto porcentaje de la muestra que tienen como autopercepción: "sin necesidad" de tratamiento en un $90,3 \%$ (tabla 1) ligeramente menor a los estudios de Crespo en Ecuador que halló un 91,18\% (5), y mayores que los estudios de Campoverde $81.30 \%$ (2), Manccini $88,6 \%$ (6), Ricse con 78,2\% (7), Bernabé 87,2\% (8), Kumar 73,6\% (9), y Morales 74,6\% (10). Mientras que un estudio realizado por Janoševiç (11), con 190 estudiantes de Serbia entre 11 a 14 años, sólo muestra un $60,4 \%$, en muchos investigaciones previas se observa que cuando el promedio de edad se encuentra 
en la preadolescencia, tienen una autopercepción mayor de no necesitar el tratamiento de ortodoncia, esto quizás se deba a que aún no tengan bien definida la autopercepción de la estética facial y bucal por la poca preocupación de los estudiantes de su aspecto estético a edades tempranas, mostrando más bien un denotado interés por construir interrelaciones personales con sus compañeros, por otro lado, puede deberse también a que, una necesidad manifiesta clínicamente percibida por el profesional no sea percibido de esa manera por los estudiantes; es más, en la valoración de la autopercepción se muestran fotos frontales intraorales que no son representativas de todas las posibilidades de malposición dentaria, no se valoran en otros planos del espacio los problemas ortodonticos que pudiera tener el estudiante, quizás por ello es que los resultados muestran que los estudiantes no perciben el problema completo; por ello existen estudios que bajo esta premisa realizan el muestreo en estudiantes jóvenes porque supuestamente es más fácil analizar el impacto social autopercibido de los trastornos bucales en adultos jóvenes, debido a que estos ya tienen cierta estabilidad emocional y una visión más realista de la estética dental-facial; sin embargo esto no es del todo cierto ya que desde un punto de vista psicológico los niños presentan una conceptualización de su estética desde los 10 años; otros autores manifiestan que la inquietud por la estética oral comienza alrededor de $\operatorname{los} 8$ años, cuando los niños empiezan a tener un criterio similar a la de los adultos en la percepción de la imagen corporal de ellos mismos (12); investigaciones futuras podrían enfocarse a grupos etarios únicamente adolescentes, jóvenes o adultos jóvenes para poder contrastar los resultados

La necesidad de tratamiento "media o moderada" fue de $4,4 \%$ del total de la muestra y un $5,3 \%$ de "necesidad definitiva" de tratamiento ortodontico, coincidente con otros estudios como los de Morales 5,6\% (10), Ngom et al., con 8,7\% (13), y Uguncu et al., $4.8 \%$ (14), mientras que el estudio realizado en Serbia por Janoševiḉ(11) no guarda concordancia con nuestro estudio, encontró un $15,3 \%$ de necesidad definida de tratamiento ortodontico, cabe mencionar que esta investigación hace mención que sus resultados son similares a algunos otros países europeos, además menciona que una vez obtenida su población de estudio evidenció que muchos de los estudiantes ya habían recibido tratamiento ortodontico en un 33\% por lo que fueron excluidos de su estudio, lo que hace notar es que gran parte de su población (en Serbia) quizás estén más involucrados en tratar de manera temprana los problemas de maloclusión a diferencia de otros países incluso de mismo Europa, esto también explicaría porque en sus resultados hay $15,3 \%$ de su muestra que tiene necesidad definida de tratamiento ortodontico, es decir están más concientizados e involucrados para poder evaluar de manera más detallada el aspecto de sus dientes y su estética facial y poder buscar tratamiento.

Al comparar el tipo de escuela pública o privada no existe diferencia significativa entre ambas $(\mathrm{p}=0.078)$ (tabla 2), existe similar autopercepción de no necesitar tratamiento ortodóntico, siendo casi un $90 \%$, mientras que la necesidad definitiva de tratamiento ortodontico fue sólo un 5\%, en ambas escuelas, estos hallazgos son similares con los encontrados por Campoverde en Ecuador, que al tratar de hallar alguna relación entre el nivel socioeconómico y su autopercepción no hallan relación alguna, puede deberse a los criterios de inclusión y exclusión de este estudio que haya homogenizado la muestra

En cuanto al sexo no hubo diferencia estadísticamente significativa $(\mathrm{p}=0,062) \quad($ tabla 3$)$, nuestro grupo muestral estuvo compuesta de un 58,5\% de género femenino y un $41,5 \%$ de género masculino, de los cuales se observa que en general en ambos géneros el $90 \%$ respondió que no necesitan tratamiento; sin embargo, fue mayor en las mujeres siendo un 53\% mientras en varones fue de $37 \%$, esto probablemente se deba a que ellas tienen mayor preocupación por el aspecto estético de su rostro y el cabello, se maquillan, disminuyendo el impacto de la maloclusión en el rostro como lo menciona Campoverde (8)(2), pese a que las mujeres tienen mayor preocupación por su aspecto facial, la menarca, el desajuste hormonal y el estrés al que se ven sometidas las mujeres jóvenes (17)(11), no perciben tener necesidad de tratamiento ortodóntico En cuanto al grupo etario tampoco se evidencia diferencia significativa ( $\mathrm{p}$-valor $=0,069)$ (tabla 4$)$ se puede evidenciar que la mayoría, respondieron que no requerían tratamiento, un detalle es que en los más jóvenes lo hicieron en mayor porcentaje 57,9\% frente a un $34,3 \%$, que no coincide con otros estudios que manifiestan que a más edad pueden tener mayor preocupación por su aspecto y por lo tanto están más conscientes en detalles que los más jóvenes no perciben, Crespo (5) halló un 91,8\% en un grupo de niños de 11 a 12 años

Bernabé et al., obtuvieron un 1,8\% del INTO-CE, al determinar los niveles de necesidad influenciados por la edad, sexo y nivel socioeconómico, no encontró asociación estadísticamente significativa 
necesariamente socioeconómicos, de edad o sexo (8). En cuanto a la variable calidad de vida podemos apreciar (tabla 5) que en general la media fue de 54,2; por lo tanto se encuentra en el nivel bajo un $50,31 \%$ y un $49,69 \%$ con una calidad alta; siendo algo relativamente mayor al estudio de Campoverde 53,65 (2)que también lo hizo en una provincia de Ecuador, pero menor al estudio de Ricse en Perú con una media de 59 (7), Thiruvenkadam en la India obtuvo una media de 59 (15), este valor algo más bajo al igual que Campoverde puede deberse a que en la población rural haya valores menores como lo señala Li (16), por tener menos accesibilidad a tratarse ortodonticamente, ya que puede tener menores oportunidades en comparación con las grandes ciudades como por ejemplo, no existe de manera directa por parte del estado una atención especializada para problemas ortodónticos en los diferentes niveles de atención del Ministerio de Salud y por otro lado la posibilidad de tratarse satisfactoriamente aquellos que están en ciudades más grandes.

En cuanto al subocomponente salud oral de la encuesta de calidad de vida (tabla 6) se obtuvo un promedio de 14,6; coincidente con los estudios de, Li 14,4 (16) y Ricse en Lima 15 (7) pero menores que Campoverde 12,87 (2) y por Broder 13,6 (17); para el subcomponente bienestar funcional se obtuvo una media de 12.5 siendo similar al estudio de Broder (17), mientras que resultó más alto al estudio de Campoverde (2) con 12,1, y más bajo que Li (16) con 13,7 y Ricse (7) en Lima 13. En cuanto al componente Bienestar Social y Emocional la media obtenida de 27.1 resulta menor a las obtenidas por Campoverde (2) en Ecuador con 28,77 en la China por Li (16) con 34.2 y Ricse (7)en Lima 31, aunque es mayor a la encontrada por Broder (17)con 26.9; en un estudio realizado en Lima por Velasquez (18) donde utiliza otro índice para medir la calidad de vida ( CPQ 1114), encuentra que los componentes de bienestar emocional y componente social tenían una puntuación más alta que correspondía a tener más afectación de dichos componentes por lo que señala que podría atribuirse a que los niños con maloclusión suelen sufrir burlas por parte de sus compañeros de clase debido a afecciones bucales visibles

La relación existente entre calidad de vida y sexo, (tabla 7) si encontramos significancia estadística con un $p=0,009$ donde el sexo femenino presenta una calidad de vida más baja en relación a los varones, siendo un $58,5 \%$ vs un $41,5 \%$ de los varones, comparando con otras investigaciones encontramos que si hay coincidencia con los estudios de Campoverde y $\mathrm{Li}$ aunque con diferencias mínimas entre ambos sexos, pese a que en la autopercepción de necesidad de tratamiento ortodontico (CE) no lo expresen, esto puede deberse a que las mujeres tienen una exigencia mayor en la belleza

Al relacionar la calidad de vida de acuerdo a las escuelas de donde provienen (tabla 8), no se hallaron diferencias significativas estadísticamente con un $p=0,073$, pese a que la mayor cantidad de muestra fue en la escuela pública, esto se relaciona con la autopercepción del componente $\mathrm{CE}$ en donde señalaron en su gran mayoría ambos grupos no necesitar un tratamiento ortodóntico; al parecer ambos grupos son influenciados de la misma manera lo que hace notar la homogeneidad de nuestra muestra

Al establecer la relación entre la necesidad de tratamiento Ortodóntico y la calidad de vida (tabla 9), se encontró relación estadísticamente significativa de acuerdo al Rho de spearman siendo de $-0,635$ y un nivel $\mathrm{p}=0,023$ que determina que existe relación entre la calidad de vida y la necesidad de tratamiento ortodontico, quiere decir que se entiende que los niños con poca necesidad de tratamiento de ortodoncia tienen una mejor calidad de vida, y aquellos con maloclusión tienen afectación de la autoestima con un promedio de calidad de vida menor

\section{CONCLUSIONES}

Existe correlación negativa moderada entre la calidad de vida y la necesidad de tratamiento ortodóntico en escolares de 12 a 15 años de dos unidades educativas público y privada de la ciudad de Huancayo 2020.

La calidad de vida en ambas escuelas fue nivel "bajo" en un 50,3\%, siendo en la escuela pública $41,2 \%$ y en la escuela privada $9,1 \%$, no se halló diferencia estadísticamente significativa entre ambos grupos de escolares, según el COHIP-SF 19.

La calidad de vida según sexo fue de un nivel "bajo" en 50,3\%, siendo en el sexo femenino $33 \%$ y en el masculino $17,3 \%$, habiendo una diferencia estadísticamente significativa entre ambos sexos, según el COHIP-SF 19.

La calidad de vida según edad fue "alta" en $52.7 \%$ para los menores de 13 años y de $26,2 \%$ para los mayores e iguales de 13 años; "media" 10,4\% para los 
menores de 13 años y de $10.1 \%$ para los mayores de 13 años y "baja" $0,6 \%$ para los menores de 13 años y 0,0\% para los mayores de 13 años. según COHIP_SF 19

La necesidad de tratamiento a través del componente estético (CE) en las escuelas fue mayoritariamente "sin necesidad de tratamiento", siendo en la escuela pública $73,6 \%$ de "sin necesidad de tratamiento", $3,2 \%$ de "moderada necesidad" y $4,4 \%$ de "necesidad definitiva", no hallándose diferencia estadísticamente significativa entre ambos tipos de escuelas.

La necesidad de tratamiento a través de su componente estético (CE) de acuerdo al género fue en el sexo femenino 53,3\% en el sexo masculino $37 \%$ "sin necesidad" de tratamiento; "necesidad moderada" en el sexo femenino fue $2,5 \% \mathrm{y}$ masculino $1,9 \%$; y con "necesidad definitiva" de tratamiento fue en el sexo femenino $2,8 \%$ y en el masculino $2,5 \%$, no hallándose diferencia estadísticamente significativa entre ambos grupos.

La necesidad de tratamiento a través de su componente estético (CE) de acuerdo a la edad fue en el grupo etario menores de 13 años $57.9 \%$ "sin necesidad", 2,8\% "necesidad moderada" y 3,8\% "necesidad definitiva"; y en el grupo etario de mayores e iguales de 13 años fue de $32,4 \%$ "sin necesidad", $1,6 \%$ "necesidad moderada" y de 2,2\% "necesidad definitiva".

\section{Correspondencia:}

Pablo Santiago Bonilla Cairo

Correo electrónico: pabsanti@hotmail.com

\section{REFERENCIAS BIBLIOGRÁFICAS}

1. Graber L. Ortodoncia principios y técnicas actuales. 6ta Edición. Barcelona, España: Editorial Elsevier; 2018.

2. Campoverde C. Necesidad de tratamiento ortodóntico y su relación con la calidad de vida relacionada a la salud oral en escolares de 12 a 15 años, de dos unidades educativas, pública y privada de la ciudad de Loja- Ecuador 2016: Tesis para optar el grado de Maestro en Estomatología. Lima, Perú: Universidad Peruana Cayetano Heredia; 2017.

3. De Oliveira CM, Sheiham A. Tratamiento de ortodoncia y su impacto en la calidad de vida relacionada con la salud bucal en adolescentes brasileños. J Orthod 2004; 31: 20-7.

4. Feiou. L. et al. Effect of personality on oral healthrelated quality of life in undergraduates. Angle
Orthod. 2018; 88:215-220.

5. Crespo C, Dominguez C, Vallejo F, et al. Impacto de maloclusiones sobre la calidad de vida y necesidad de tratamiento ortodóntico en escolares de dos escuelas privadas Azogues - Ecuador, 2015. Rev Estomatol Herediana. 2017;27(3):141-52. DOI: https://doi.org/10.20453/reh.v27i3.3198

6. Manccini LM. Necesidad de tratamiento ortodóntico según el índice de necesidad de tratamiento ortodóntico en escolares de 12 a 13 años. Tesis para obtener título profesional de Cirujano Dentista. Lima, Perú: Universidad Nacional Mayor de San Marcos; 2011.

7. Ricse E. Impacto de las condiciones orales atribuidas a maloclusiones sobre la calidad de vida y la necesidad de tratamiento ortodóntico en escolares de 11 a 12 años de un colegio de un distrito urbano, San Juan de Lurigancho. Tesis de Maestría en Estomatología. Lima, Perú: Universidad Peruana Cayetano Heredia; 2011.

8. Bernabé E, Flores C. Normative and self-perceived orthodontic treatment need of a Peruvian university population. Head Face Med. 2006; 3: 2-22.

9. Kumar P, Londhe B, Kotwal A, Mitra R. Prevalence of malocclusion and orthodontic treatment need in school children an epidemiological study. MJAFI. 2013; 6: 369-37.

10. Morales D. Prevalencia de maloclusiones y necesidad de tratamiento ortodóntico (OITN), en escolares con dentición mixta distrito de Ate Vitarte en el año 2015. Tesis para optar el título de cirujano dentista. Lima Peru: Universidad de ciencias aplicadas; 2016.

11. Janoševiḉ P, Stošiḉ M, Janoševiḉ M, Radojiçicḉ J, Filipoviḉ G, Ḉutoviḉ T. Index of orthodontic treatment need in children from the Niš Region. Vojnosanit Pregl. 2015; 72(1):12-5.

12. Auria-Martin B, Retamar-Jimenez S, Sanz-Corasa A. A. Afectación de la maloclusión en la calidad de vida del paciente odontopediátrico. RCOE. 2018;23(2): 43-50.

13. Ngom P, Diagne F, Dieye F, Diop-Ba K, Thiam F. Orthodontic Treatment Need and Demand in Senegalese School Children Aged 12-13 Years. The Angle Orthodontist. 2007; 77(2): 323-30.

14. Uguncu N, Ertugay E. The use of the Index of Orthodontic Treatment Need (IOTN) in a School Population and Referred Population. J Orthod. 2001; 28 (1): 45-52.

15. Thiruvenkadam G, Asokan S, John B, Priya G, Prthiba J. Oral health-related quality of Life of children seeking orthodontic treatment based on child oral health impact profile: a cross-sectional study. Contemp Clin Dent. 2015; 6(3):396-400.

16. Li Ch, Xia B, Wang Y, Guan X, Yuan J, Ge L. Translation and psychometric properties of the Chinese (Mandarin) version of the Child Oral Health 
Impact Profile-Short Form 19 (COHIP-SF 19) for school-age children. Health Quality Life Outcomes. 2014; 12: 3 .

17. Broder H, Wilson-Genderson M. Reability and convergent and discriminatvalidity of child oral health impact profile (COHIP SF-19). J Public Health Dent. 2012; 72(4): 302-12.
18. Velasquez O. Relación entre la necesidad de tratamiento ortodóntico y calidad de vida en adolescentes y sus familias. Instituto Nacional de Salud del Niño. Tesis para optar el título de segunda especialidad profesional en odontopediatría. Lima Perú: Universidad nacional mayor de San Marcos; 2020 .

Recibido: 10/02/2021

Aceptado: 29/05/2021 